\title{
Penetrating Injuries to the Stomach, Duodenum, and Small Bowel
}

\author{
Jordan A. Weinberg • Martin A. Croce
}

Published online: 8 April 2015

(C) Springer International Publishing AG 2015

\begin{abstract}
Since operative management became the standard for hollow viscus injury, the techniques regarding the identification and subsequent repair of injuries to the stomach, small intestine, and duodenum have remained relatively unchanged. In this manuscript, we describe the contemporary management of penetrating hollow viscus injury as practiced at the Elvis Presley Memorial Trauma Center in Memphis, Tennessee. We commence with the general principles of laparotomy for trauma, followed by specific discussion of the practice and pitfalls of the operative management of gastric, duodenal, and small intestinal wounds.
\end{abstract}

Keywords Penetrating trauma · Gastric injury · Duodenal injury $\cdot$ Small bowel injury

\section{Introduction}

Although the care of the injured patient has evolved considerably over the past century, the principles of treatment of penetrating hollow viscus injuries remain remarkably similar. Since operative management became the standard for hollow viscus injury, the techniques regarding the identification and subsequent repair of injuries to the stomach, small intestine, and duodenum have remained relatively unchanged. Only the

This article is part of the Topical Collection on Penetrating Injuries to Hollow Abdominal Viscera

J. A. Weinberg $(\bowtie) \cdot$ M. A. Croce

Department of Surgery, University of Tennessee Health Science

Center, 910 Madison Ave, 2nd Floor, Memphis, TN 38163, USA

e-mail: jaw@uthsc.edu

M. A. Croce

e-mail: mcroce@uthsc.edu application of the surgical stapler or the "damage control" approach would seem foreign to the general surgeons of the 1940s; otherwise, they would easily recognize the conduct of laparotomy for penetrating abdominal injury in 2015. In this manuscript, we describe the contemporary management of penetrating injuries to the stomach, small intestine, and duodenum as practiced at the Elvis Presley Memorial Trauma Center in Memphis, Tennessee. The approach to diagnostic workup of penetrating abdominal injury is left for another discussion. Herein, we will focus on the conduct of the laparotomy in general and the specific management of injuries.

\section{General Principles}

Prior to skin incision, antibiotics with broad-spectrum aerobic and anaerobic coverage should be administered. If hollow viscus injury is confirmed, the antibiotics should be continued for no more than $24 \mathrm{~h}$. Data from prospective studies clearly indicate that prolonging antibiotic administration beyond $24 \mathrm{~h}$ provides no additional protection against surgical site infection $[1 \bullet \bullet]$. In patients admitted with hemorrhagic shock, the administered dose of antibiotics may be increased twofold or threefold and repeated after transfusion of every 10 units of blood.

Abdominal exploration is performed through a generous midline incision to permit evaluation of the entire peritoneal cavity. Once initial control of any significant bleeding has been obtained, the next step is to control any contamination from spilled gastrointestinal contents. Babcock clamps are useful for temporarily controlling contamination from bowel perforations without causing injury to the bowel wall. Management of specific injuries is outlined in the next sections.

On completion of the operation, the abdominal wall fascia is typically closed with running monofilament absorbable suture. Handling of the skin (primary closure vs. gauze packing 
and closure by secondary intention) is a consideration that should not be taken lightly. In fasting patients, the stomach harbors low numbers of bacteria because of its low $\mathrm{pH}$. Trauma patients, however, often arrive with full stomachs, and a more neutral $\mathrm{pH}$ and a higher bacterial count in the leaking stomach content can be expected. If a gastric perforation is encountered, we recommend that secondary or delayed primary skin closure should be performed in view of the increased risk of surgical site infection. This is particularly true when significant hemorrhage or associated colon injury is present, in which case the rate of intra-abdominal abscess formation may be as high as $24 \%$ [2]. The incidence of wound complications from isolated small bowel injury is relatively low $(6 \%$ in a single institution study [3]), and most surgeons recommend proceeding with primary closure in this scenario. We prefer to err on the side of caution and will tend to leave the skin open to heal by secondary intention for such bowel injuries, reserving primary closure for cases where minimal bowel contamination is encountered.

The patient with severe abdominal injuries may benefit from abbreviated laparotomy. This approach has evolved from a realization that the critically injured patient often requires rapid, lifesaving bailout techniques to arrest hemorrhage and control enteric spillage to preempt the combined physiologic insult of the trauma and surgery from progressing to irreversible shock and death [4]. With respect to hollow viscus injuries managed in this scenario, reconstruction of intestinal continuity (anastomosis) following bowel resection is not performed at the initial operation and is instead deferred to subsequent laparotomy following resuscitation of the patient (typically 24 to $48 \mathrm{~h}$ following the initial operation). The damage control approach is employed to prevent or mitigate the development of acidosis, coagulopathy, and/or hypothermia secondary to major blood loss and resuscitation. This decision should be made early in the operative course and be based on the rapid recognition of injury patterns rather than the onset of physiologic derangement, which may herald the onset of irreversible shock.

\section{Injuries to the Stomach}

Assessment of the stomach for injury commences with full visualization of its anterior surface from the pylorus to the esophagogastric junction. The posterior surface of the stomach is exposed by opening the gastrocolic ligament, which provides approach to the lesser sac. On opening the gastrocolic ligament, injury to the vascular arcade of the greater curvature should be avoided. With the stomach elevated superiorly, the transverse colon is retracted inferiorly to expose the posterior gastric wall. Adhesions between the posterior gastric wall and the pancreas, if present, should be carefully divided to provide full exposure. Grasping the stomach can be assisted by advancement of a nasogastric tube along the greater curvature. The nasogastric tube can then function as a handle to hold the stomach. Care must be taken to avoid placing excess tension on the greater curvature of the stomach and the short gastric vessels, thus avoiding iatrogenic injury to the spleen. The greater and lesser curvatures of the stomach should be closely inspected because the fatty omental attachments may obscure an underlying gastric wound. Such inspection is particularly important in the setting of a small-caliber missile or stab wound. The perforation can be remarkably small, and the serosal tissue damage in such cases is often subtle (particularly in the setting of obesity). Additionally, although a stab wound may result in a solitary gastric perforation, gunshot wounds through hollow organs usually result in even numbers of full-thickness injuries, given the propensity of the missile to pass through and through the injured organ. The finding of an odd number of wounds shot prompt repeat inspection of the stomach for occult injury.

Repair of gastric wounds is dictated by their severity, classified according to the grading system of the American Association for the Surgery of Trauma (AAST) (Table 1). Intramural hematomas (grade I) are managed by unroofing and evacuation of the hematoma, followed by seromuscular closure with interrupted silk suture. Most gastric perforations are of grades II or III and are manageable with primary repair. Given the tendency of the well-vascularized gastric wall to bleed at the site of injury, a two-layer technique is recommended to secure hemostasis. When the wound involves the pylorus, incorporation of the wound with a pyloroplasty prevents stenosis. Suture repair of wounds at the cardioesophageal junction may be reinforced by gastric fundoplication.

More extensive wounds are not amenable to primary repair (grades IV and V). Such injuries include significant tissue loss

Table 1 Stomach injury scale

\begin{tabular}{lll}
\hline Grade $^{\mathrm{a}}$ & Description of injury & AIS-90 \\
\hline I & Contusion/hematoma & 2 \\
& Partial-thickness laceration & 2 \\
II & Laceration $<2 \mathrm{~cm}$ in GE junction or pylorus & 3 \\
& $<5 \mathrm{~cm}$ in proximal $1 / 3$ stomach & 3 \\
& $>10 \mathrm{~cm}$ in distal $2 / 3$ stomach & 3 \\
III & Laceration $>2 \mathrm{~cm}$ in GE junction or pylorus & 3 \\
& $>5 \mathrm{~cm}$ in proximal $1 / 3$ stomach & 3 \\
& $>10 \mathrm{~cm}$ in distal $2 / 3$ stomach & 3 \\
IV & Tissue loss or devascularization $<2 / 3$ stomach & 4 \\
V & Tissue loss or devascularization $>2 / 3$ stomach & 4 \\
\hline
\end{tabular}

$G E$ gastroesophageal

${ }^{\text {a }}$ Advance one grade for multiple lesions up to grade III. Used with permission from Trauma and Acute Care Surgery, The American Association for the Surgery of Trauma. I have obtained permission and have the form in my office 
and/or devascularization and often are associated with major vascular injury as a consequence of the proximity of the major vessels and the force necessary to cause such a significant injury. Patients with these injuries often do not survive to hospital, so these extensive gastric wounds are rarely encountered. Grade IV injuries can usually be managed by means of a partial gastrectomy. Restoration of gastrointestinal continuity is accomplished with either a gastroduodenostomy or a gastrojejunostomy. In the exceedingly rare event of complete gastric devascularization or destruction, a total gastrectomy would be required, but we have yet to encounter this scenario.

\section{Injuries to Small Intestine}

The small intestine is assessed by "running the bowel." The small intestine and its mesentery are inspected from the ligament of Treitz to the ileocecal valve. If active mesenteric bleeding is encountered, it is controlled by isolation and individual ligation of the injured vessels rather than by mass suture ligation of the mesentery, which may result in bowel ischemia. As bowel perforations are identified, temporary control measures, such as the application of Babcock clamps, may be performed in an effort to prevent excessive or ongoing soilage. Low velocity injuries, such as knife wounds or lowcaliber gunshot wounds, may be subtle, particularly at the mesenteric border of the small intestine, so close inspection of even the smallest hematomas is advocated when running the bowel.

Management of each wound is determined by its severity according to the AAST grading system (Table 2). Partialthickness injuries (grade I) are managed by closing the seromuscular defect with simple sutures. Small full-

Table 2 Small bowel injury scale

\begin{tabular}{|c|c|c|c|}
\hline Grade $^{\mathrm{a}}$ & $\begin{array}{l}\text { Type of } \\
\text { injury }\end{array}$ & Description of injury & AIS-90 \\
\hline \multirow[t]{2}{*}{ I } & Hematoma & $\begin{array}{l}\text { Contusion or hematoma } \\
\text { without devascularization }\end{array}$ & 2 \\
\hline & Laceration & Partial thickness, no perforation & 2 \\
\hline II & Laceration & Laceration $<50 \%$ of circumference & 3 \\
\hline III & Laceration & $\begin{array}{l}\text { Laceration } \geq 50 \% \text { of circumference } \\
\text { without transection }\end{array}$ & 3 \\
\hline IV & Laceration & Transection of the small bowel & 4 \\
\hline \multirow[t]{2}{*}{ V } & Laceration & $\begin{array}{l}\text { Transection of the small bowel } \\
\text { with segmental tissue loss }\end{array}$ & 4 \\
\hline & Vascular & Devascularized segment & 4 \\
\hline
\end{tabular}

${ }^{a}$ Advance one grade for multiple injuries up to grade III. Used with permission from Trauma and Acute Care Surgery, The American Association for the Surgery of Trauma. I have obtained permission and have the form in my office thickness wounds (grade II) are repaired with limited debridement (if necessary) and closure. Closure is performed in either one or two layers (we prefer a single-layer closure), and transverse closure is recommended to avoid luminal narrowing. Larger full-thickness wounds (grade III) may be repaired primarily in transverse orientation, providing that luminal narrowing can be avoided. Otherwise, resection and anastomosis should be performed. Extensive wounds and wounds associated with devascularization (grades IV and V) are treated with resection and anastomosis. Once all bowel injuries are accounted for, the decision must be made whether to perform primary repairs, resections, or some combination of the two. Primary repair of multiple low-grade injuries preserves bowel length and is generally preferred. At the discretion of the operating surgeon, resection of a segment containing multiple injuries may be performed to expedite the operation and minimize suture lines, provided that the amount of bowel to be resected is small enough that its loss would not have any effect on digestive function. As discussed for gastric injuries, an odd number of gunshot wounds would be the exception to the rule and should prompt further inspection of the bowel for missing injury.

When mesenteric injury is encountered in the absence of bowel injury, the adjacent bowel must be closely assessed for evidence of vascular compromise. If the bowel appears viable, the rent in the mesentery should be sutured closed (avoiding mesenteric vessel ligation) after bleeding is controlled to prevent an internal hernia. If there is evidence of vascular compromise, bowel resection and anastomosis are indicated.

Determination of intestinal viability begins with assessment of the bowel's appearance. Adjunctive measures, such as the use of a handheld Doppler device or fluorescein infusion with Wood lamp illumination, may help if bowel viability is equivocal. We prefer to use a handheld Doppler device because it is easy to use and is readily available in our operating rooms. Audible Doppler signal at the antimesenteric side of the bowel wall confirms the presence of arterial flow, which reliably verifies bowel viability.

Small bowel anastomoses are usually handsewn in one or two layers, although stapling devices may also be used. Handsewn techniques are generally favored by our group, but the choice of technique depends largely on the surgeon's preference. A multicenter retrospective study of trauma laparotomies suggested that stapled anastomoses had a higher complication rate than did sutured anastomoses. Overall, $13 \%$ of stapled anastomoses were associated with an intraabdominal postoperative complication, compared with $5 \%$ of sutured anastomoses [5]. As this study did not separate small intestinal anastomoses from colonic anastomoses, it is unclear to what extent the results apply specifically to small bowel anastomoses. It is likely, however, that bowel edema contributes to staple line failure. If bowel edema is evident or anticipated, it may be wise to perform a sutured anastomosis. 


\section{Injuries to the Duodenum}

The duodenum is relatively small compared to the other hollow viscera, and therefore less likely to be injured in the event of penetrating trauma. Nonetheless, injury to the duodenum, relative to the stomach or small bowel, is more complicated and fraught with risk, given the close anatomic association to the pancreas, common bile duct, and major vascular structures. In addition, the relative infrequency with which duodenal injuries are encountered has resulted in a less than robust reported clinical experience from which to draw.

To thoroughly explore the duodenum for wounds, it is important to consider the anatomic divisions of the duodenum and the surrounding tissues that may mask the presence of injury. The first portion of the duodenum is partially obscured by the edge of the gastroduodenal ligament, and full exposure of the first portion often benefits from partial division of this ligament at its attachment to the duodenum. The second portion of the duodenum is a retroperitoneal structure and requires the Kocher maneuver for full exposure. Ideally, a complete Kocher maneuver results in visualization of the left renal vein. The third portion of the duodenum is obscured by the superior mesenteric vessels, and dissection of the mesenteric attachments to the duodenal wall is necessary to fully expose the third portion. The fourth portion of the duodenum is partially obscured by the ligament of Treitz. Releasing the ligament of Treitz allows for full exposure of the fourth portion.

Stab wounds to the duodenum are uncommon, given the relatively deep location of the duodenum, but when encountered are typically located on the anterior surface of the duodenum and occasionally on the posterior surface as a result of a stab wound to the patient's back. Nonetheless, it is important to fully mobilize the duodenum to inspect all sides for the possibility of a through-and-through injury. Gunshot wounds are the more common variety of duodenal wounds. Often, a single wound will be initially encountered, and a second wound may not be initially seen. As stressed earlier, it is important to remember that almost all gunshot wounds through bowel result in through-and-through injuries, and chalking up a single wound to a tangential trajectory may result in a missed injury. This underlies the importance of fully mobilizing and examining the duodenum as described above prior to declaring a single tangential wound, as the partner wound may be hidden in the duodenum's retroperitoneal attachments. A particularly difficult location to examine is the area of apposition between the duodenum and pancreas. Should only a single wound be appreciable following the customary duodenal mobilization techniques, it is prudent to expose the inside of the duodenum by extending the opening of the existing woundthereby the inside of the duodenum in the area of pancreatic apposition may be evaluated for the partner wound.

Management of duodenal wounds, once fully exposed and accounted for, may be guided by the severity of injury as outlined by the AAST grading system (Table 3). Partialthickness duodenal wounds are not commonly encountered as a result of penetrating mechanism. They may be simply oversewn with interrupted seromuscular sutures. Fullthickness wounds, provided they can be closed without tension or narrowing of the duodenum, can be managed with suture repair alone. Compared to the other hollow viscus organs, breakdown of the duodenal suture line and formation of a fistula occurs with relative frequency, up to $33 \%$ in a recent study [6•]. Multiple surgical adjunctive measures to prevent this dreaded complication have been advocated. Herein, the approach that our group has developed over time for the management of these wounds is described.

For relatively simple duodenal wounds (AAST grade 2), suture repair alone generally suffices. Simple wounds would be characterized more specifically as stab wounds, low velocity gunshot wounds, and wounds that do not involve the portion of the duodenum that apposes the pancreas. Leaving a periduodenal drain as a safety measure to control a duodenal fistula, should the suture line breakdown is not recommended; the foreign body effect of the drain may in fact favor fistula formation [7]. We will leave a closed suction drain, however, for control of an associated pancreatic head injury (see below).

For the relatively more complex duodenal wound, it is our recommendation that decompression of the duodenal suture line(s) be accomplished by the placement of a retrograde tube

Table 3 Duodenum injury scale

\begin{tabular}{llll}
\hline Grade $^{\text {a }}$ & $\begin{array}{l}\text { Type of } \\
\text { injury }\end{array}$ & Description of injury & AIS-90 \\
\hline I & Hematoma & $\begin{array}{l}\text { Involving single portion of } \\
\text { duodenum }\end{array}$ & 2 \\
II & $\begin{array}{l}\text { Laceration } \\
\text { Pematoma }\end{array}$ & $\begin{array}{l}\text { Involving more than one portion } \\
\text { Disruption }<50 \% \text { of circumference }\end{array}$ & 2 \\
III & $\begin{array}{l}\text { Laceration } \\
\text { Laceration }\end{array}$ & $\begin{array}{c}\text { Disruption 50-75 \% of } \\
\text { circumference of D2 } \\
\text { Disruption 50-100 \% of circumference } \\
\text { of D1, D3, and D4 }\end{array}$ & 4 \\
IV & Laceration & $\begin{array}{c}\text { Disruption >75 \% of circumference } \\
\text { of D2 }\end{array}$ & 5 \\
& & $\begin{array}{c}\text { Involving ampulla or distal } \\
\text { common bile duct } \\
\text { Massive disruption of } \\
\text { duodenopancreatic complex }\end{array}$ & 5 \\
V & Laceration & 5 \\
\hline
\end{tabular}

$D 1$ first position of duodenum, $D 2$ second portion of duodenum, $D 3$ third portion of duodenum, $D 4$ fourth portion of duodenum

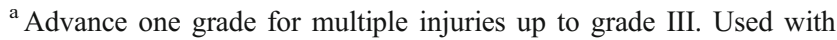
permission from Trauma and Acute Care Surgery, The American Association for the Surgery of Trauma. I have obtained permission and have the form in my office 
jejunostomy. Specifically, a red rubber catheter is placed in the proximal jejunum using a Witzel technique and threaded retrograde into the duodenum. The tube is placed to gravity drainage, thus decompressing the suture line. A second tube jejunostomy is placed just distal to the first, in the standard antegrade direction to be used for enteral feeding in the early postoperative period, and for long-term feeding access should a duodenal complication develop. This procedure has become known as the twin jejunostomy (Fig. 1). When first described, a third tube (Stamm gastrostomy) was advocated (the socalled triple tube decompression), but we prefer to use nasogastric tube decompression of the stomach as an alternative. Once the patient exhibits return of bowel function and no signs of intra-abdominal sepsis, the retrograde tube is clamped and then the nasogastric tube is sequentially discontinued, followed by introduction of oral intake. We do not routinely perform contrast studies to assess for healing prior to initiation of oral intake.

Duodenal decompression may also be achieved by placement of a tube duodenostomy rather than a retrograde jejunosotmy. This technique in effect creates a controlled duodenal fistula that should theoretically close as the tract matures, and the drain is progressively withdrawn. Unfortunately, the fistula may persist, so this technique is no longer recommended.

Pyloric exclusion is an alternative technique that remains in contemporary use. The goal of pyloric exclusion is to protect the duodenal suture line by diverting gastric contents away from the duodenum. Described for trauma by Vaughan et al. in 1977, the procedure involves the performance of a gastrostomy, followed by purse-string suture closure of the pylorus [8]. Over time (usually by 4 weeks), the muscular pylorus will reopen, even with the use of nonabsorbable suture. Pyloric closure can similarly be accomplished with the application of a noncutting stapling device on the pylorus, but

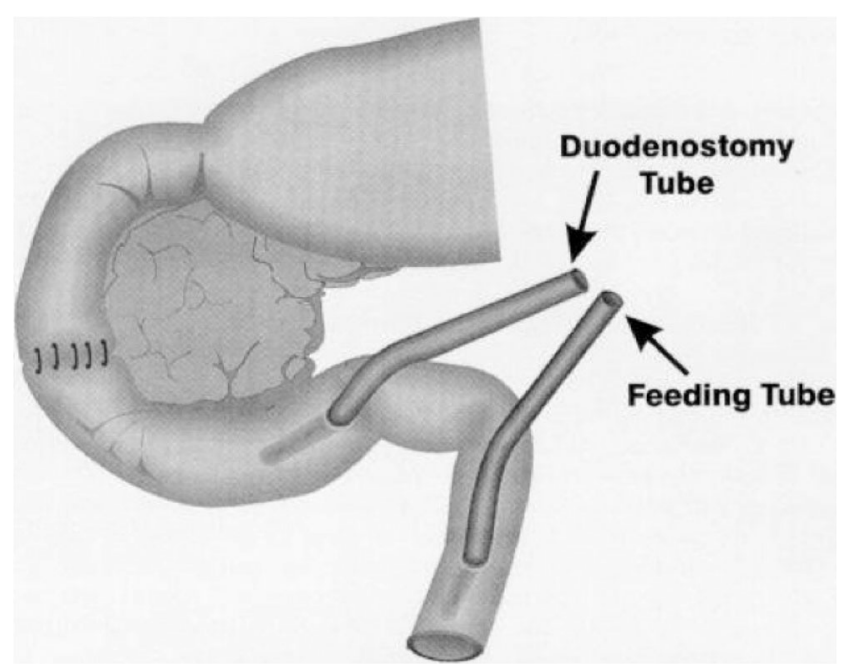

Fig. 1 Twin jejunostomies. Retrograde jejunostomy is depicted as Duodenostomy Tube and antegrade jejunostomy as Feeding Tube we prefer purse-string suture of the pylorus. A gastrojejunostomy is performed to restore gastrointestinal continuity. Conceptually, the duodenum will heal, while the pylorus remains closed. Once the pylorus reopens, the gastrointestinal stream should resume its normal course, and the gastrojejunostomy functionally closes. We have occasionally used pyloric exclusion as an adjunct for particularly complex duodenal wounds, especially wounds involving the medial wall of the second portion. Since those wounds can be difficult to adequately close, pyloric exclusion with twin jejunostomies are valuable adjuncts for these particular wounds.

Reported experience with both the twin jejunostomy technique and pyloric exclusion has been mixed. Stone and Fabian reported their experience with the twin jejunostomy (actually the triple tube technique) in 1979 [7]. They observed that the addition of internal decompression reduced the duodenal suture line leak rate from 8 to less than $1 / 2 \%$. Conversely, others have not been able to reproduce these outcomes and in fact reported worse outcomes associated with tube decompression and recommend against their use. A similar mixed experience has been associated with pyloric exclusion $[9,10 \bullet]$. Recently, the philosophy of "less is more" has been advocated for duodenal injuries. Talving et al. reported a $3 \%$ duodenal fistula rate among duodenal gunshot wound patients managed repair or resection alone [11]. More recently, Ordonez et al. reported their experience with complex penetrating duodenal injuries also treated without adjunctive techniques [6॰]. Although their duodenal related mortality rate was relatively low $(<3 \%)$, the number of duodenal fistula was quite high (33\%), suggesting that repair or resection alone may not be the best choice for all injuries.

The close proximity of the duodenum to the pancreas and bile duct makes for a high likelihood of combined injuries, particularly for wounds involving the second portion of the duodenum. Most injuries involving the duodenum and head of the pancreas can be managed with repair of the duodenum (plus twin jejunostomies) and closed suction drainage of the pancreas to control leakage from the injured pancreas. On occasion, the surgeon will be faced with an extensive duodenal injury that precludes repair or resection. If the pancreas is largely intact, the duodenum may be debrided, followed by a Roux-en-Y reconstruction with the Roux limb sewn to create an anastomosis with the opening in the duodenum. Alternatively, jejunum can be used as a serosal patch over the duodenal opening. Extensive destruction of both the duodenum and pancreatic head, however, may likely be best managed with pancreaticoduodenectomy. The ampulla should be clearly identified (by inspection of the duodenal mucosa through the wound), both to assess for injury and to avoid iatrogenic injury during repair of the duodenal wall. Concern for periampullary injury should prompt a cholangiogram to assess for distal common bile duct injury. In the event of a combined injury to the common bile duct, pancreas, and duodenum, 
pancreaticoduodenectomy (i.e., Whipple procedure) should be strongly considered (the alternative would be to repair the duodenum, drain the pancreatic injury, and divert the bile by way of cholecystojejunostomy or choledochojejunostomy provided the duct is relatively generous in size). Reconstruction of the gastrointestinal tract following pancreaticoduodenectomy is accomplished with gastrojejunostomy. A generous pancreatic duct allows for duct to mucosa pancreaticojejunostomy; however, most trauma patients have a relatively small duct, leaving dunking pancreaticojejunostomy or duct ligation without anastomosis as options. Similarly, a relatively small common bile duct may be better managed with ligation and cholecystojejunostomy, provided the cystic duct is patent and gallbladder appears normal. Such devastating injuries to the pancreaticoduodenal complex are often associated with severe blood loss, necessitating a staged approach, whereby bleeding is controlled and the pancreaticoduodenal resection is completed at the initial laparotomy, and reconstruction is performed at subsequent laparotomy following resuscitation and correction of hypothermia and coagulopathy.

\section{Conclusion}

The contemporary management of penetrating injuries to the stomach, small intestine, and duodenum as practiced at the Elvis Presley Memorial Trauma Center includes the systematic assessment of the hollow organs for injury. Care must be taken to thoroughly inspect the gastric curvatures and the mesenteric border of the small bowel, as these are areas where full-thickness injuries may be obscured. Additionally, full mobilization of the duodenum as described above is necessary to avoid missed duodenal injury (resulting in subsequent severe and potentially fatal abdominal sepsis). An odd number of gunshot wounds should prompt thorough reinspection for a missing injury, as gunshot wounds tend to result in an even number of wounds. Management of relatively small gastric and small intestinal wounds can usually be accomplished by primary repair, with resection and anastomosis reserved for more extensive wounds. Management of duodenal injuries is relatively more complex, given the relation of the duodenum of the pancreas and common bile duct. Although simple duodenal wounds may be managed with primary repair alone, we recommend that the treatment of more complex wounds be augmented by the twin jejunostomy technique as described. Strong consideration should be given to performing pancreaticoduodenectomy when a periampullary injury has resulted in combined injuries of the duodenum, pancreas, and common bile duct.

\section{Compliance with Ethics Guidelines}

Conflict of Interest Jordan A. Weinberg and Martin A. Croce declare no conflict of interest.

Human and Animal Rights and Informed Consent This article does not contain any studies with human or animal subjects performed by any of the authors.

\section{References}

Papers of particular interest, published recently, have been highlighted as:

- Of importance

•• Of major importance

1.• Goldberg SR, Anand RJ, Como JJ, et al. Prophylactic antibiotic use in penetrating abdominal trauma: an Eastern Association for the Surgery of Trauma practice management guideline. J Trauma Acute Care Surg. 2012;73:S321-5. Evidence-based practice guideline for effective prophylactic antibiotic use in penetrating abdominal trauma, updated in 2012.

2. Croce MA, Fabian TC, Patton Jr JH, et al. Impact of stomach and colon injuries on intra-abdominal abscess and the synergistic effect of hemorrhage and associated injury. J Trauma. 1998;45: 649.

3. Salim A, Teixeira PG, Inaba K, et al. Analysis of 178 penetrating stomach and small bowel injuries. World J Surg. 2008;32:471-5.

4. Hirshberg A, Walden R. Damage control for abdominal trauma. Surg Clin N Am. 1997;77:813.

5. Brundage SI, Jurkovich GJ, Hoyt DB, et al. Stapled versus sutured gastrointestinal anastomoses in the trauma patient: a multicenter trial. J Trauma. 2001;51:1054.

6. Ordonez C, Garcia A, Parra MW, et al. Complex penetrating duodenal injuries: less is better. J Trauma Acute Care Surg. 2014;76: $1177-83$. Recently published study advocating the "less is more" approach to the operative management of duodenal injuries.

7. Stone HH, Fabian TC. Management of duodenal wounds. J Trauma. 1979;19:334-9.

8. Vaughan III GD, Frazier OH, Graham DY, et al. The use of pyloric exclusion in the management of severe duodenal injuries. Am J Surg. 1977;134:785-90.

9. Cogbill TH, Moore EE, Feliciano DV, et al. Conservative management of duodenal trauma: a multicenter perspective. J Trauma. 1990;30:1469-75.

10. Dubose JJ, Inaba K, Teixeira PG, et al. Pyloric exclusion in the treatment of severe duodenal injuries: results from the National Trauma Data Bank. Am Surg. 2008;74:925-9. Evaluation of duodenal injuries from a national database. Using propensity scoring, the authors demonstrated that pyloric exclusion conferred no outcome or survival benefit.

11. Talving P, Nicol AJ, Navsaria PH. Civilian duodenal gunshot wounds: surgical management made simpler. World J Surg. 2006;30:488-94. 